\title{
Tpl2 regulates intestinal myofibroblast HGF release to suppress colitis-associated tumorigenesis
}

\author{
Vasiliki Koliaraki, Manolis Roulis, and George Kollias \\ Institute of Immunology, Biomedical Sciences Research Centre (BSRC) "Alexander Fleming”, Vari, Attica, Greece.
}

\begin{abstract}
The tumor microenvironment plays a significant role in colitis-associated cancer (CAC). Intestinal myofibroblasts (IMFs) are cells in the intestinal lamina propria secreting factors that are known to modulate carcinogenesis; however, the physiological role of IMFs and signaling pathways influencing CAC have remained unknown. Tumor progression locus $2(\mathrm{~T} p 12)$ is a MAPK that regulates inflammatory and oncogenic pathways. In this study we addressed the role of Tpl2 in CAC using complete and tissue-specific ablation of $\mathrm{Tpl} 2$ in mutant mice. Tpl2-deficient mice did not exhibit significant differences in inflammatory burdens following azoxymethane (AOM)/dextran sodium sulfate (DSS) administration compared with wild-type mice; however, the mutant mice developed significantly increased numbers and sizes of tumors, associated with enhanced epithelial proliferation and decreased apoptosis. Cell-specific ablation of Tpl2 in IMFs, but not in intestinal epithelial or myeloid cells, conferred a similar susceptibility to adenocarcinoma formation. Tpl2-deficient IMFs upregulated HGF production and became less sensitive to the negative regulation of HGF by TGF- $\beta 3$. In vivo inhibition of HGF-mediated c-Met activation blocked early, enhanced colon dysplasia in Tpl2-deficient mice, indicating that Tpl2 normally suppresses the HGF/c-Met pathway. These findings establish a mesenchyme-specific role for $\mathrm{T} p 12$ in the regulation of HGF production and suppression of epithelial tumorigenesis.
\end{abstract}

\section{Introduction}

Colorectal cancer (CRC) and colitis-associated cancer (CAC), a subtype of CRC associated with inflammatory bowel disease (IBD), are major health risks and the second most common cause of cancer mortality in Western societies. Tumorigenesis in CRC and $\mathrm{CAC}$ is thought to evolve via heterotypic interactions between cancer cells and multiple recruited or resident stromal cell types forming the tumor microenvironment. A stromal component of particular importance to tumorigenesis, especially in CAC, has been the inflammatory component, which appears to promote the neoplastic potential of intestinal epithelial cells (IECs) via the production of proinflammatory cytokines and chemokines, proangiogenic and growth factors, ROS, and proinvasive matrixdegrading proteases $(1,2)$. A multitude of studies on the azoxymethane (AOM)/dextran sodium sulfate (DSS) animal model of CAC have highlighted several inflammatory pathways modulating the development of colorectal tumors. It has been shown that both NF-KB and the IL-6-activated JAK/STAT3 pathways are protumorigenic, functioning via both immune cell modulation and IEC survival and proliferation (3). In contrast, a tumor-suppressive role has been demonstrated for the inflammasome/IL-18/IL-18R/ myeloid differentiation factor 88 (MYD88) axis, in the absence of which AOM/DSS-treated mice show impaired tissue repair and excessive commensal bacteria-driven inflammation and tumorigenesis $(4,5)$. Despite, however, the extensive evidence on specific signaling pathways underlying inflammatory cell recruitment and their effector functions during tumorigenesis $(3,4,6)$, relatively little is known about the molecular mechanisms mediating resident stromal cell activation and crosstalk with the adjacent tumor epithelium and its microenvironment.

Conflict of interest: The authors have declared that no conflict of interest exists. Citation for this article: J Clin Invest. 2012;122(11):4231-4242. doi:10.1172/JCI63917.
The intestinal subepithelial myofibroblast (IMF) is an important cell of the intestinal lamina propria known to orchestrate tissue repair, angiogenesis, inflammation, and fibrosis $(7,8)$. IMFs appear to also play an important role in intestinal mucosal morphogenesis and the formation of the epithelial stem cell niche by secreting factors such as noncanonical Wnts, BMPs, and various antagonists, as well as extracellular matrix, that together control stem cell growth $(9,10)$. More recently, compelling evidence was presented of a role for IMFs in establishing and maintaining colon cancer stem cell (CSC) fate through the secretion of HGF and consequent regulation of epithelial Wnt signaling (11). Interestingly, activation of paracrine HGF signaling was identified as a potential mechanism to explain the oncogenic potential of loss of TGF- $\beta$ responsiveness in fibroblasts from prostate and squamous cell carcinoma of the forestomach (12). Moreover, conditional ablation of tumor suppressor PTEN from mammary stromal fibroblasts was shown to activate ETS2-mediated transcription via downregulation of microRNA-320, promoting mammary epithelial tumor angiogenesis and invasion $(13,14)$. It appears, therefore, that stromal fibroblasts including IMFs perform critical modulatory functions in setting the milieu for the onset and expansion of epithelial tumors. However, cell-specific signaling pathways of physiological significance for the homeostasis and effector functions of IMFs in CRC are still missing.

Tpl2 is a serine-threonine kinase that belongs to the family of mitogen-activated protein kinase kinase kinases (MAP3Ks) and is known to exert both inflammatory and oncogenic functions (15). It acts mainly by regulating the activation of the MAPK ERK, through direct phosphorylation of its MAPK MEK. It is activated by a variety of signals, including TLR ligands, TNF, IL- $1 \beta$, and CD40 ligand (CD40L), and is able to transduce additional downstream signaling pathways that are stimulus- and cell type-specific, including JNK, p38, ERK5, and NF-KB (16). Initial evidence 
A

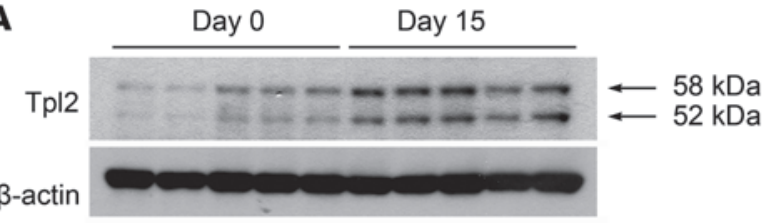

B

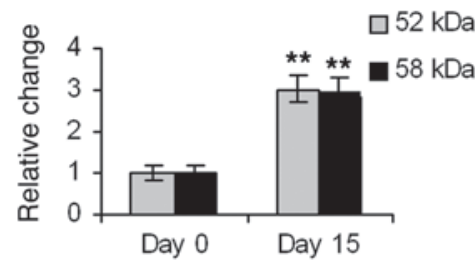

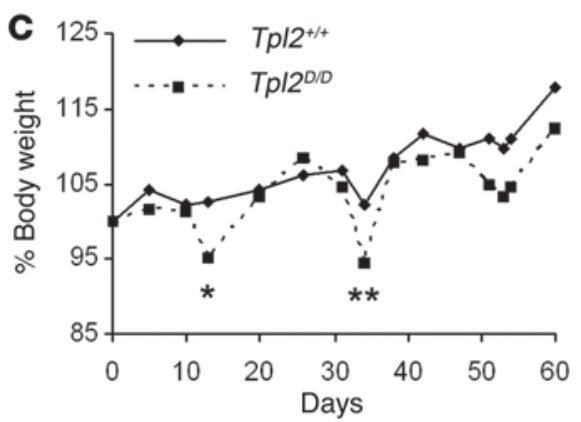

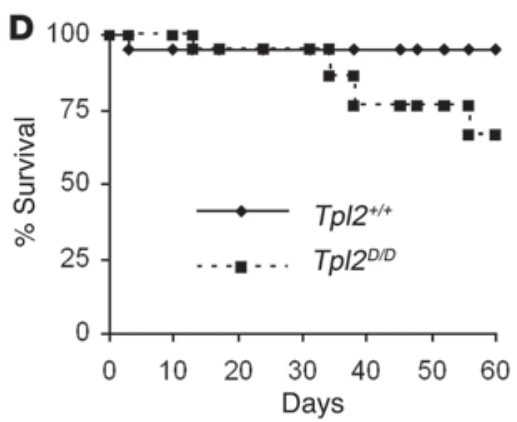

$\mathbf{E}$
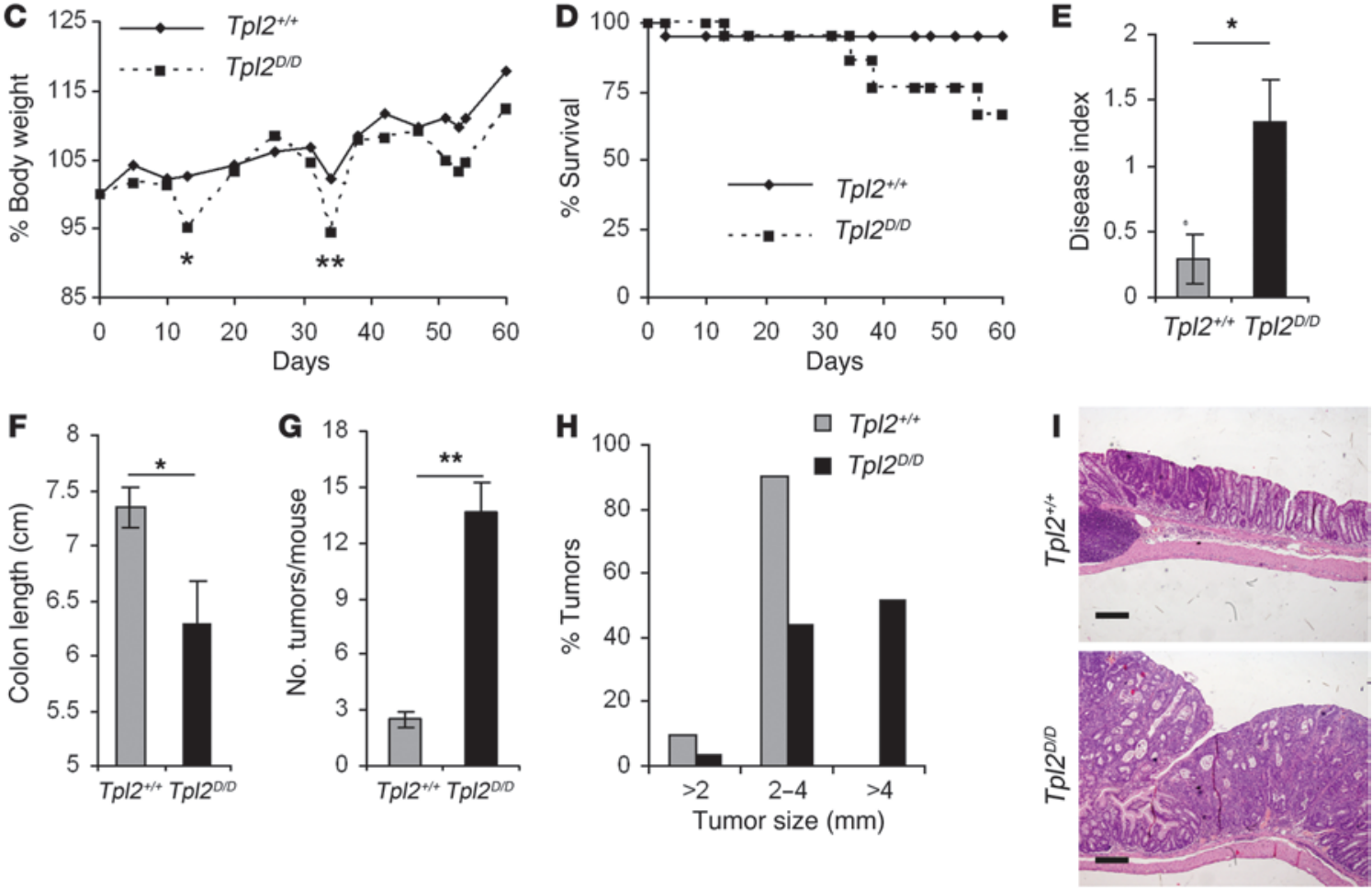

$\mathbf{J}$

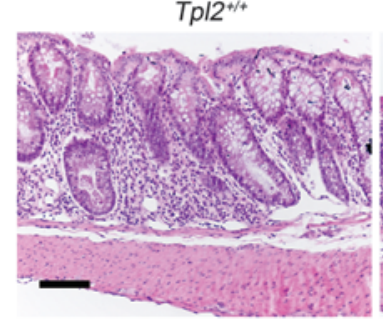

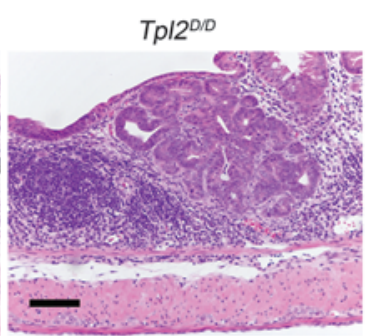

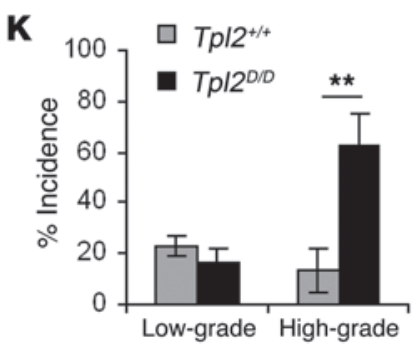

Figure 1

Tp/2D/D mice display increased tumorigenesis upon AOM/DSS administration. Western blot of Tpl2 expression on days 0 and 15 of the AOM/DSS regime $(\mathbf{A})$ and densitometric analysis $(\mathbf{B})$ of both isoforms of Tpl2 protein $(52 \mathrm{kDa}$ and $58 \mathrm{kDa})$ relative to $\beta$-actin. Data represent mean $\pm \mathrm{SEM}$. $n=5$; ${ }^{\star *} P<0.01$. Tp/2D/D mice and wild-type littermate controls were subjected to the AOM/DSS model of colitis-associated colon carcinogenesis. Body weight changes (C) and survival rates (D) were monitored during the course of the experimental procedure. (E) Disease index was measured on day 13 after initial AOM injection. Colon length $(\mathbf{F})$, number of tumors per mouse $(\mathbf{G})$, and size of tumors $(\mathbf{H})$ measured at the end of the experimental protocol. The data correspond to one representative experiment of three $(n=6)$, except in the case of survival curves and size distribution graphs, which are cumulative from 3 experiments. Data represent mean \pm SEM. ${ }^{*} P<0.05,{ }^{* *} P<0.01$. (I) Representative images of formalin-fixed and H\&E-stained colon sections at the end of the experimental protocol (day 60). Scale bars: $200 \mu \mathrm{m}$. (J) Representative images of formalin-fixed and H\&E-stained colon sections early (day 15) during the experimental protocol. Scale bars: $50 \mu \mathrm{m}$. (K) Dysplasia incidence was measured at an early time point, 5 days after the end of the first DSS cycle. The data shown correspond to the average of 4 experiments performed $(n=6)$. Data represent mean \pm SEM. ${ }^{* *} P<0.01$.

using Tpl2-knockout mice showed that it has a proinflammatory role mainly by controlling myeloid cell secretion of important inflammatory mediators such as TNF, IL-6, COX-2, $\mathrm{PGE}_{2}$, and IL-10 (17-19). Concerning the tumorigenic properties of Tpl2, this kinase was first identified as a target gene for provirus inser- tion in MoMuLV-induced T cell lymphomas and MMTV-induced mammary adenocarcinomas, where a truncated form of the protein was shown to be responsible for its procarcinogenic properties (20-22). However, although a tumor-promoting role for Tpl2 has been implied in virus-related malignancies and in breast cancer 
$(21,23,24)$, there is evidence that it may act as a tumor suppressor, as shown in Tpl2-deficient mice developing enhanced lymphomas in the MHC class I-restricted T cell antigen receptor (TCR) transgenic background (25) and a higher number and incidence of chemically induced skin tumors (26). So far, there is very little evidence on the role of the Tpl2 kinase in intestinal inflammation and carcinogenesis. Early studies have shown increased expression of Tpl2 in colon cancer cell lines (27), while evidence from our laboratory has revealed a protective role for $\mathrm{T} p 12$ in intestinal inflammation (28). More recently, a milder form of acute DSSinduced colitis was indicated in Tpl2-deficient mice, without, however, significant alterations of inflammatory cell infiltration and cytokine release (29).

In this study we have addressed the role of Tpl2 in the AOM/ DSS model of chronic CAC using complete and tissue-specific ablation of $\mathrm{Tpl} 2$ in mutant mice. Our findings establish a novel intestinal fibroblast-specific role for $\mathrm{Tpl} 2$ in the regulation of HGF production and the prevention of epithelial tumors in CAC

\section{Results}

Tpl2-deficient mice are highly susceptible to colitis-induced colorectal carcinogenesis. To investigate Tpl2-mediated mechanisms in inflammationinduced colorectal carcinogenesis, we used the well-established AOM/DSS mouse model of CAC (Supplemental Figure 1A; supplemental material available online with this article; doi:10.1172/ JCI63917DS1). We initially searched for possible alterations in the expression levels of Tpl2 during CAC. Western blotting of total lysates from mouse colon indicated increased expression of Tpl2 early during AOM/DSS administration (Figure 1, A and B). This finding is in line with the upregulated $\mathrm{T} p 12$ recently evidenced in human colon adenocarcinomas (30). Immunohistochemical analysis showed Tpl2 staining in epithelial cells and other cells in the mucosa, which was enhanced following AOM/DSS treatment (Supplemental Figure 1B). The muscle layer and immune cells in lymphoid aggregates did not show positive staining.

To decipher Tpl 2 function in CAC, we used a recently developed strain of conditional Tpl2-knockout mice (our unpublished observations), generated following the same targeting strategy described previously by Dumitru et al. (17). Tlp $2^{D / D}$ mice, in which the Tlp2 gene is deleted in all cells, were born at the expected Mendelian ratio and appeared healthy and fertile. As in previous studies (17), $T p l 2^{D / D}$ mice exhibited resistance to LPS/D-galactosamine lethality and decreased production of TNF by peritoneal macrophages upon stimulation with LPS (Supplemental Figure 2). When Tpl2 $2^{D / D}$ mice were subjected to the AOM/DSS model of CAC, they displayed increased body weight loss specifically at the recovery stage after DSS cycles and decreased survival in comparison to wildtype littermate controls (Figure 1, C and D). In correlation with the observed weight loss following the first DSS cycle, they also exhibited an increased disease index as assessed by measurements of diarrhea and rectal bleeding scores (Figure 1E). At completion of the protocol, on day 60 after AOM injection, $T p l 2^{D / D}$ mice and wild-type controls were euthanized, colons were resected, and both colon length and tumor number were measured. Tumor incidence was $100 \%$ in both experimental groups and controls (data not shown), but $T p l 2^{D / D}$ mice displayed decreased colon length and a marked increase in the number of macroscopically visible tumors (Figure 1, F and G). Histological analysis of colon sections on day 60 after AOM treatment revealed no difference in inflammatory or tissue damage indices between the two groups (Supplemental
Figure 3). However, tumors from $T p l 2^{D / D}$ mice were significantly larger in size in comparison to those from wild-type controls, with approximately $50 \%$ of $T p l 2^{D / D}$ mice developing tumors larger than $4 \mathrm{~mm}$ in diameter (Figure 1, H and I). Moreover, histological analysis of colon sections performed on day 15 after AOM injection, an early time point during the carcinogenic process, again failed to reveal statistically significant differences in either inflammation or tissue damage scores (Supplemental Figure 3). However, even at this early time point, $T p l 2^{D / D}$ mice exhibited significantly increased incidence of high-grade dysplasia (Figure 1, J and K).

To begin to understand the mechanisms that led to increased tumor burden in the Tpl2-knockout mice, we subjected both $T p l 2^{D / D}$ and wild-type mice to another model of chemically induced CRC, consisting of repeated AOM injections in the absence of DSS. Interestingly, in this model, where tissue destruction and subsequent inflammation are absent, there were no significant differences in either tumor incidence or numbers (Supplemental Figure 4). Taken together, these data establish a tumor-suppressive role for $\mathrm{Tp} 12$ in CAC. Notably, in the absence of Tpl2, tissue disruption and subsequent inflammation caused by DSS appear crucial for the induction of enhanced colorectal tumorigenesis.

Tpl2 modulates epithelial cell proliferation and apoptosis without influencing inflammation. To further characterize the nature of the deregulated carcinogenic signals in $T p l 2^{D / D}$ mice, we examined basic parameters related to the tumorigenic process in the intestine. We found that colonic epithelial proliferation was significantly increased early during the experimental protocol, as assessed by BrdU staining (Figure 2, A and B). Moreover, apoptosis in the intestine was decreased in $T p l 2^{D / D}$ mice early during the treatment (Figure 2, A and C). These findings are in agreement with the observed increase in dysplasia incidence and tumor formation in Tpl2-deficient mice shown in Figure 1, J and K. Consistent with these data, we also found increased expression of CAC surrogate markers, such as iNOS, $\beta$-catenin, and the stem cell marker SRYbox containing gene 9 (Sox9) in Tpl2 ${ }^{D / D}$ mice early during the disease (Figure 2D). Moreover, we identified additional key molecules associated with carcinogenesis whose RNA expression was increased, such as $c-M y c$, Hif1a, WNT1-inducible signaling pathway protein 1 (Wisp1), the chemokine KC, and Igf1 (Figure 2E). We also detected alterations, albeit moderate, in the expression levels of several inflammatory markers, such as Cox2, Tnf, and monocyte chemotactic protein-1 (Mcp1), which were increased, while others, such as $I l 6, I l 1 b$, and $I l 10$, either did not show significant changes or were below the detection limit (Figure 2E). To examine cell-specific differences in the ensuing inflammatory response, we performed immunohistochemical analysis for $\mathrm{F} 4 / 80^{+}$, Gr- $1^{+}$, and $\mathrm{CD} 4^{+}$cells in the colon at both early and late stages of the experimental protocol. In all cases we were not able to detect significant differences in inflammatory cell infiltrates between $T p l 2^{D / D}$ and wild-type mice (Figure 2F). We also measured Treg numbers in the colon of wild-type and $T p l 2^{D / D}$ mice early during disease and found no statistically significant differences (Supplemental Figure 5), which agrees with our observation of the lack of difference in their inflammatory status. Taken together, these findings indicate that Tpl 2 modulates the pro-carcinogenic environment and influences epithelial cell proliferation and apoptosis downstream of tissue damage and inflammation in CAC.

Tpl2 in IMFs is required for tumor suppression in CAC. To examine the cellular basis of the tumor-suppressive role of Tpl2, we crossed mice carrying the floxed $T p l 2$ allele $\left(T p l 2^{f l f l}\right)$ with tissue- 
A

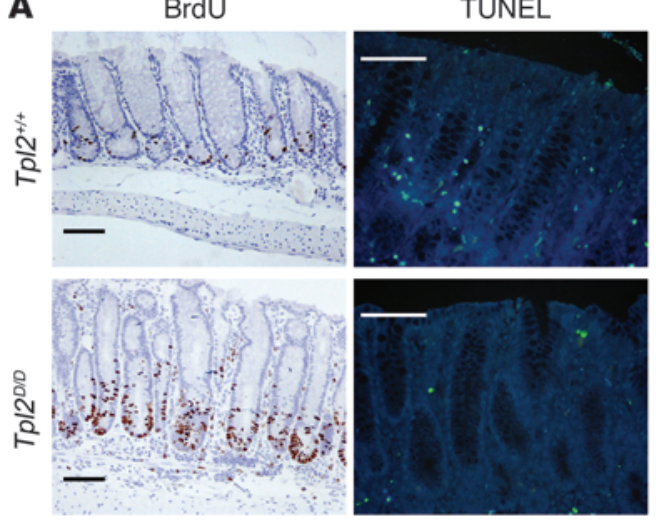

B

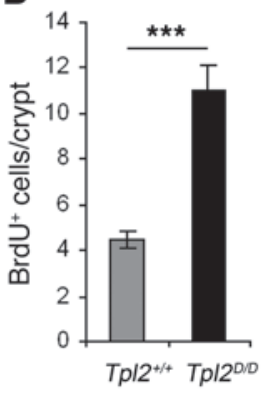

E

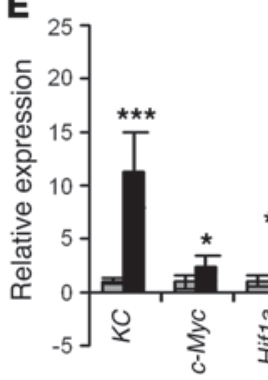

C

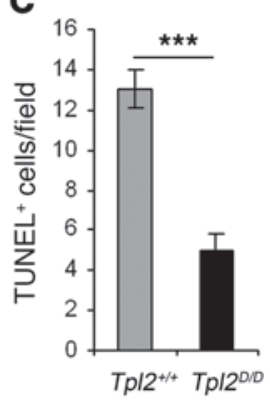

D

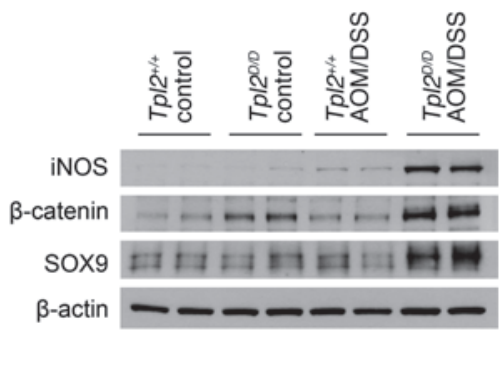

- Tp/2010

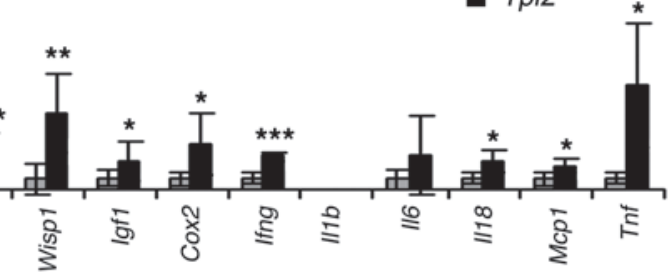

F $\quad \mathrm{F} 4 / 80$
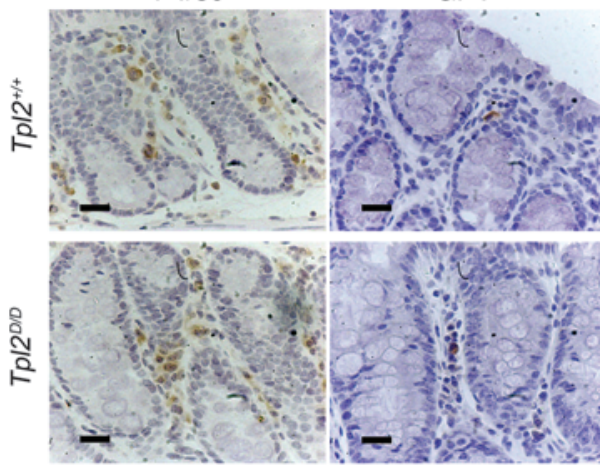

Day 15

Gr-1

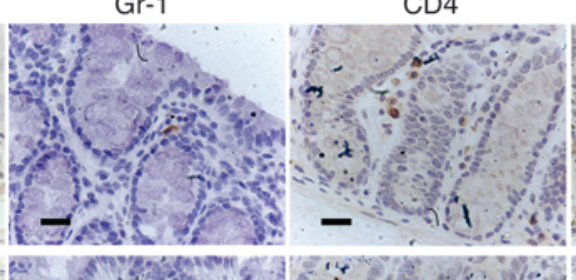

F4/80

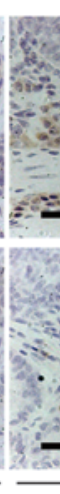

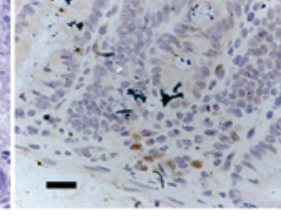

$-$
Gr-1

CD4
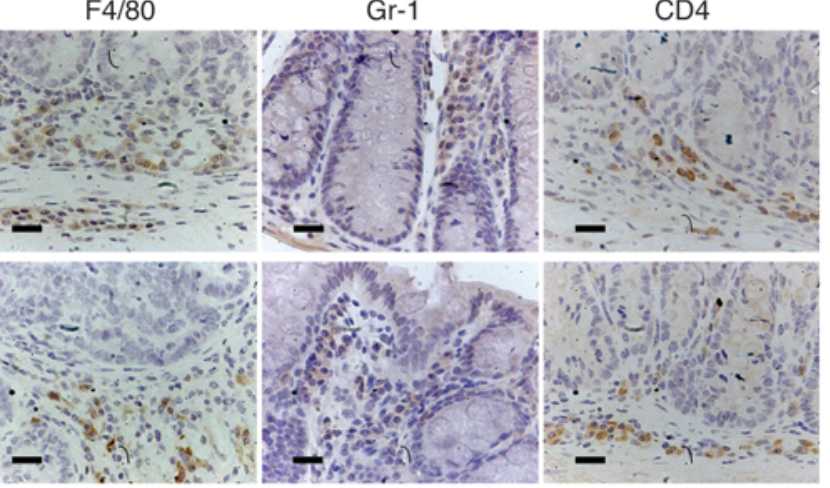

Day 60

Figure 2

$T p / 2^{D / D}$ mice display enhanced proliferation and decreased apoptosis, associated with tumorigenic protein and gene expression signatures early during the disease. (A) Formalin-fixed, paraffin-embedded colon sections from wild-type and Tp/2D/D on day 15 after AOM/DSS initiation were stained with BrdU and TUNEL assay kits for assessment of proliferation and apoptosis, respectively. Representative images are presented. Scale bars: $50 \mu \mathrm{m}$. Quantification of BrdU-positive cells per crypt (B) and TUNEL-positive cells per field (C) was performed using at least 20 random crypts and 10 random fields, respectively. Data represent mean \pm SEM. $n=6$; ${ }^{* * *} P<0.001$. (D) Western blot analysis of whole colon lysates from WT and Tp/2D/D mice on day 8 after AOM/DSS administration. Data represent one of 3 experiments performed. (E) qRT-PCR of genes of interest in whole colon of WT and Tp/2D/D mice on day 8 after AOM/DSS administration relative to untreated controls. Gene expression was normalized to $B 2 m$ levels. Data represent mean \pm SEM of 6 mice per genotype (2 mice from each of 3 individual experiments). ${ }^{*} P<0.05$, ${ }^{* *} P<0.01$, ${ }^{\star \star \star} P<0.001$. (F) Representative images from immunostaining of colon tissue samples $(n=6)$ on days 15 and 60 of the experimental protocol with antibodies against F4/80-, Gr-1-, and CD4-positive cells. Scale bars: $20 \mu \mathrm{m}$.

specific Cre-driver strains. We used lysozyme-Cre (LysM-Cre), villin-Cre, and collagen VI-Cre (ColVI-Cre) mice, respectively, to achieve cell-specific ablation of Tpl2 in myeloid cells $\left(T p l 2^{\text {myelko }}\right)$ (31), IECs (Tpl2 IECko $)(32)$, and IMFs (Tpl2 IMFko $)$ (33). Initially, we examined the efficiency of recombination in each $T p l 2$ conditional knockout mouse line by Western blot analysis (Figure 3A). We next applied the AOM/DSS protocol of CAC in all 3 mouse lines. Surprisingly, neither $T p l 2^{\text {myelloo }}$ nor $T p l 2^{I E C k o}$ mice displayed differences in the number of macroscopically visible tumors in comparison to their $T p l 2^{f f / f l}$ littermates. Notably, however, $T p l 2^{I M F k o}$ mice had significantly higher tumor burdens in comparison to controls at the end of the experimental protocol (Figure 3B). No difference in tumor incidence and numbers could be observed in ColvI-Cre mice and littermate wild-type controls that were also subjected to the AOM/DSS protocol, excluding possible off-target effects of the Cre transgene on tumor formation during CAC (Supplemental Figure 6).

Further analysis of the increased tumorigenic phenotype of $T p l 2^{I M F k o}$ mice indicated many similarities to $T p l 2^{D / D}$ mice. First, Tpl2IMFko mice showed, in addition to the increased number of tumors at the end of the protocol, a significant increase in tumor size (Figure 3, C and D). Furthermore, they showed increased 
A

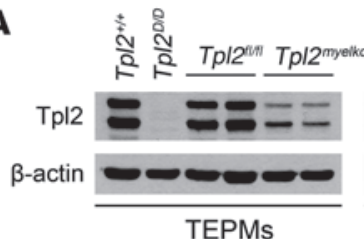

B

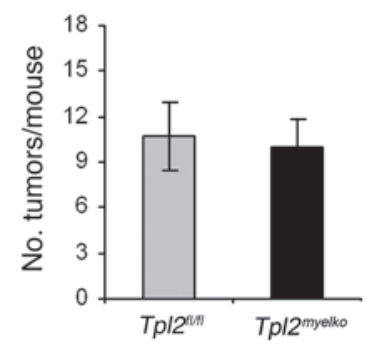

C

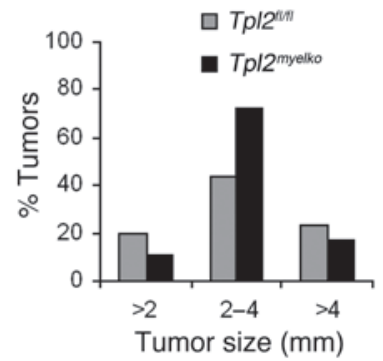

D
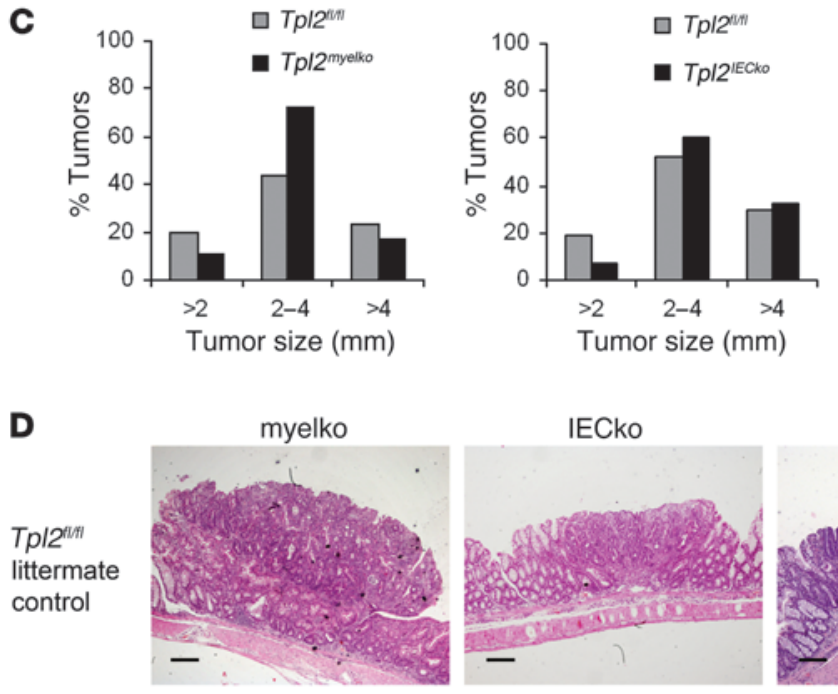
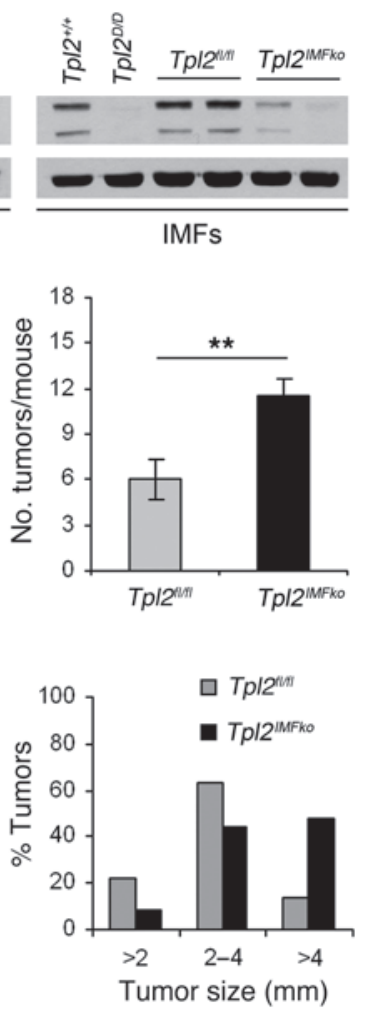

Figure 3

$T p / 2^{\text {IMFko }}$ mice, but not $T p / 2^{\text {myelko }}$ or $T p / 2^{I E C k o}$ mice, exhibit increased susceptibility to CAC. Tp/2 ${ }^{f l / f l}$ mice were crossed with LysM-Cre, villin-Cre and ColVI-Cre mice in order to accomplish cell-specific deletion of Tpl2 in myeloid cells (Tp/2myelko), IECs (Tp/2/ECko), and intestinal myofibroblasts (Tp/2 IMFko), respectively. (A) Western blot analysis in protein lysate from thioglycolate elicited peritoneal macrophages (TEPMs), IECs, and IMFs was used in each case to verify efficient deletion of Tpl2 protein. (B) These mice were subjected to the AOM/DSS protocol of CAC. Tumor multiplicity was measured in $T p / 2^{\text {myelko }}$, Tp/2IECko , and Tp/2IMFko mice in comparison to $T p / 2^{f / f l}$ littermate controls on day 60 after AOM/DSS administration. Data represent mean \pm SEM from one of 2 (for Tp/2myelko mice) or 3 (for Tp/2IECko and $T p / 2^{I M F k o}$ ) experiments performed. $n=5 ;{ }^{*} P<0.01$. (C) Size distribution of colonic tumors in Tp/2myelko, Tp/2/ECko, and Tp/2IMFko mice and their respective littermate controls at the end of the experimental protocol. The data shown are cumulative of 2 (for Tp/2myelko mice) or 3 (for Tp/2IECko and Tp/2IMFko) experiments performed. (D) Representative images from H\&E staining of colon tissues from Tp/2myelko, Tp/2IECko, and Tp/2IMFko mice and their respective littermate controls on day 60 of the regime. Scale bars: $200 \mu \mathrm{m}$.

high-grade dysplasia formation early during the disease (Figure 4, A and B), associated with increased proliferation and decreased apoptosis, as assessed by BrdU and TUNEL staining, respectively (Figure 4, A, C, and D). Examining gene expression signatures on day 15 after AOM administration, we found increased expression of genes related to tumorigenesis, such as c-Myc, Hifla, Wisp1, KC, and Igf1, though no difference could be found for most proinflammatory molecules, such as Tnf, Cox2, Il6, and Illb (Figure 4E). As in Tpl2-deficient mice, no significant differences in inflammation or tissue damage indices could be detected in Tpl2 IMFko mice either early or late during the experimental protocol (Supplemental Figure 7, A and B). Moreover, neither Tpl2 myelko nor Tpl2 $2^{I E C k o}$ mice displayed differences in tissue damage and inflammation indices (Supplemental Figure 7, A and B), epithelial proliferation, apoptosis, or cytokine expression (Supplemental Figure 8, A-D, and Supplemental Figure 9, A-D, respectively). It should also be noted that neither Tpl2myelko, Tpl2 IECko , nor Tpl2 IMFko mice showed differences in survival, weight loss, or colon length when compared with their littermate controls (Supplemental Figure 7, C and D). Furthermore, it was noted that on day 15 $T p l 2^{D / D}$ mice displayed macroscopically visible tumors in approximately $20 \%$ of the cases (data not shown), while $T p l 2^{\text {IMFko }}$ mice never showed adenoma formation at this time point. Molecular analysis was therefore performed on day 8 and day 15 for $T p l 2^{D / D}$ and Tpl2 $2^{I M F k o}$ mice, respectively, so as to best represent early time points, before tumor formation. These phenotypic differences between $T p l 2^{D / D}$ and $T p l 2^{I M F k o}$ mice could be attributed either to non-complete deletion of Tpl2 in the latter or to a possible contribution to the $T p l 2^{D / D}$ phenotype by another yet-unidentified cell type. Collectively, these data establish the importance of IMFs in CAC and a physiologically significant IMF-specific role for $\mathrm{Tpl} 2$ in the regulation of intestinal tumorigenesis. 
A

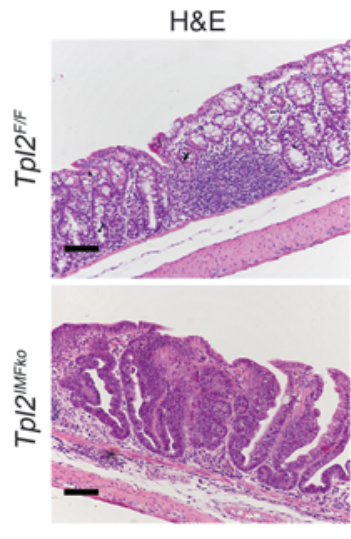

B

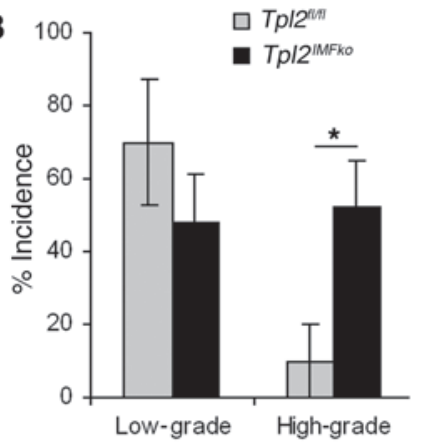

E

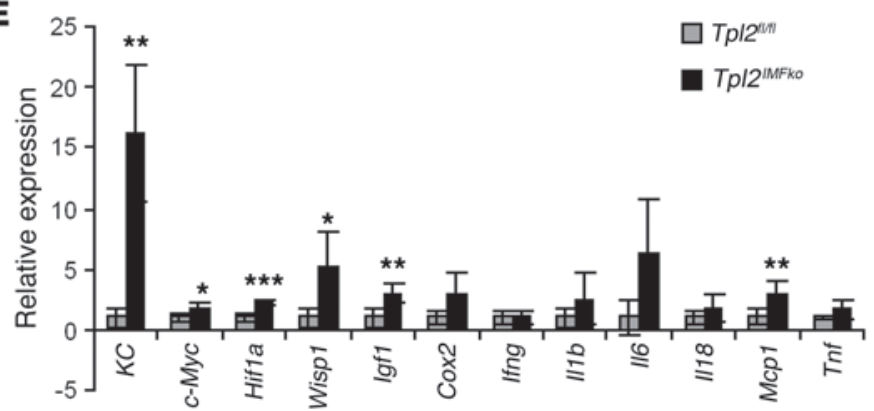

BrdU

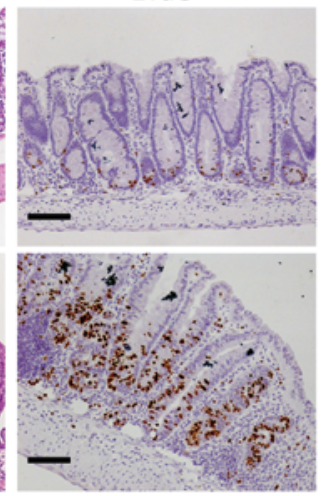

C

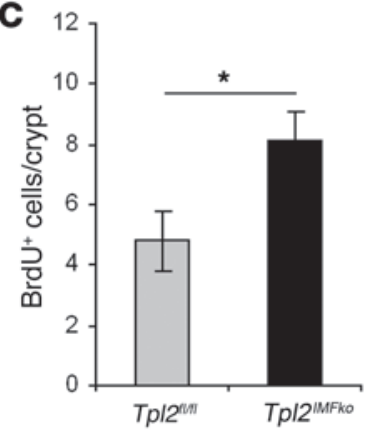

TUNEL

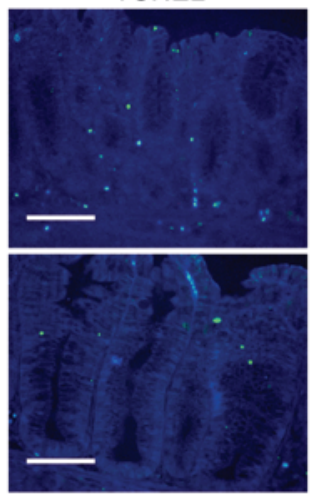

D

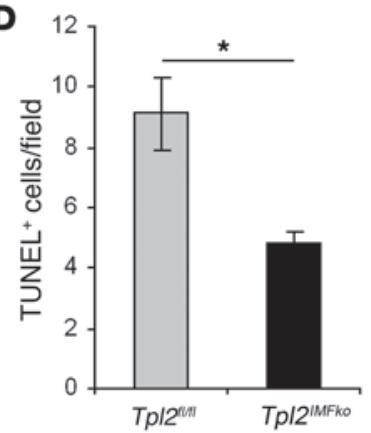

\section{Figure 4}

Tp/2IMFko mice display a pro-tumorigenic phenotype similar to $T p / 2^{D / D}$ mice. (A) Representative images from $\mathrm{H} \& \mathrm{E}, \mathrm{BrdU}$ and TUNEL staining of colon tissue slides from Tp/2/MFko and $T p / 2^{f / f l}$ mice on day 15 after AOM injection. Scale bars: $50 \mu \mathrm{m}$. (B) Dysplasia incidence was measured 15 days after the end of the AOM injection. The data correspond to the average of 4 experiments $(n=5)$. Data represent mean \pm SEM. ${ }^{*} P<0.05$. (C and $\mathbf{D}$ ) Proliferation and apoptosis as determined by measuring BrdU-positive cells per crypt (C) and TUNEL-positive cells per field (D) in colon tissue from Tp/2 IMFko and $T p / 2^{f / f f}$ mice on day 15 after AOM injection. At least 20 random crypts and 10 random fields were used, respectively. Data represent mean \pm SEM. $n=6$; ${ }^{\star} P<0.05$. (E) qRT-PCR of colon tissue on day 15 of the experimental procedure relative to samples from untreated controls. Gene expression was normalized to $B 2 m$ levels. Data represent mean \pm SEM of 6 mice per genotype (2 mice from each of 3 individual experiments). ${ }^{\star} P<0.05$, ${ }^{* \star} P<0.01,{ }^{* * *} P<0.001$.
Tpl2 regulates the TGF- $\beta 3 / H G F$ axis in IMFs. IMFs are known to modulate gut inflammation and cancer mainly through the production and secretion of soluble factors that are able to affect proliferation, apoptosis, and differentiation of adjacent epithelial cells. Such molecules include chemokines, cytokines, matrix metalloproteinases, and growth factors $(7,34)$. Interestingly, quantitative real-time PCR (qRT-PCR) of colon tissue indicated a significant increase in Hgf expression in AOM/ DSS-treated Tpl2 $2^{D / D}$ mice early during the disease (Figure 5A). HGF is a growth factor that is mainly produced by fibroblasts and acts on adjacent epithelial cells, which express its receptor, the oncogene c-Met; this leads to its phosphorylation and the subsequent activation of multiple signaling pathways, including PI3K/Akt, Wnts, and MAPKs $(11,35)$. Consistent with the observed increase in $H g f$ production, Western blots from whole colon lysates revealed increased phosphorylation levels of c-Met, as well as Akt, an important downstream target of c-Met, in AOM/DSS-treated Tpl2 $2^{D / D}$ mice (Figure 5B). Similarly, Tpl2 IMFko mice also displayed increased expression of HGF, along with increased c-Met and Akt phosphorylation in comparison to
$T p l 2^{f l f l}$ littermates early during the experimental protocol (Figure 5, C and D). It should be noted here that neither Tpl2myelko nor Tpl2 ${ }^{I E C k o}$ showed any difference in HGF production (Supplemental Figure 7E and Supplemental Figure 8E, respectively). To further define the origin and target of HGF, we isolated epithelial cells and stroma from $T p l 2^{D / D}$ mice and wild-type controls at an early stage during the experimental protocol and verified that the stromal compartment is the one that predominantly expresses HGF in the mouse colon, while epithelial cells are the responders to HGF signals (Figure 5, E and F).

To dissect the biochemistry of the tumor-suppressive function of Tpl2 in IMFs, we isolated these cells from the mouse colon of $T p l 2^{D / D}$ mice and found that Tpl2-deficient IMFs showed increased spontaneous secretion of HGF (Figure 6A). The importance of HGF secretion for adjacent epithelial cell transformation has been well studied in the TGF- $\beta$ pathway context. Bhowmick et al. (12) reported that mice lacking TGF- $\beta$ RII in fibroblasts develop spontaneous adenocarcinomas in the prostate and forestomach, due to increased HGF secretion. TGF- $\beta$ signaling is an important regulator of HGF production, and reduced HGF secretion has been reported 


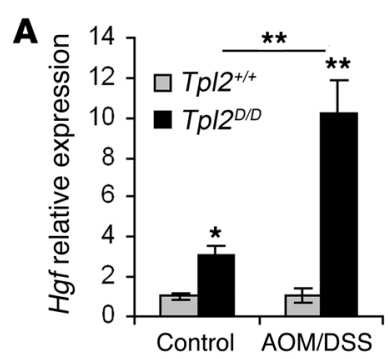

B

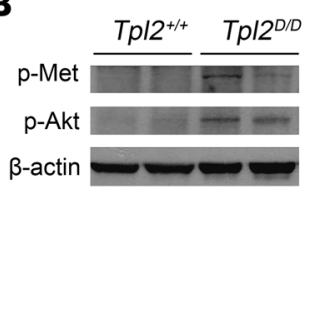

$\mathbf{E}$

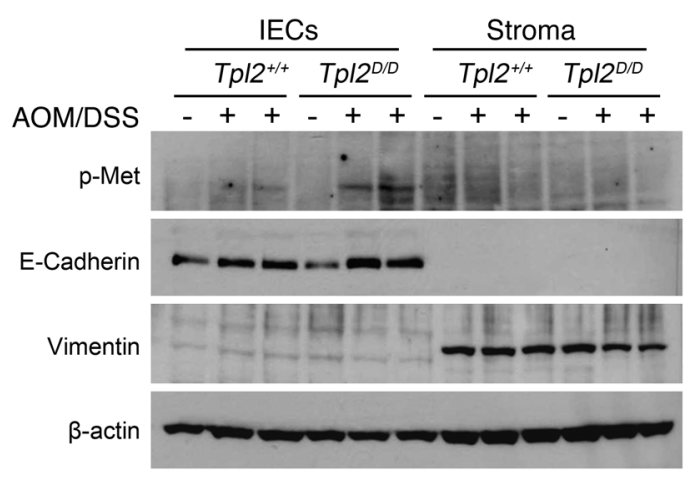

C

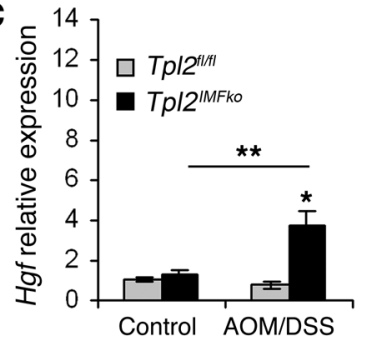

$\mathbf{F}$

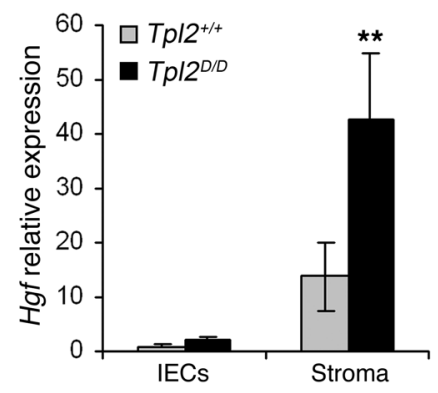

Figure 5

Tpl2 ablation results in increased HGF expression in IMFs and subsequent activation of the c-Met signaling pathway in IECs. (A) Hgf gene expression levels in colons from wild-type and Tp/2D/D mice on days 0 and 8 of the AOM/DSS experimental protocol. Gene expression was normalized to $B 2 m$ levels. Data represent mean \pm SEM of 6 mice per genotype (2 mice from each of 3 individual experiments). ${ }^{* *} P<0.01$. (B) Western blot analysis of colon lysates from the same mice for $p$-Met and $p$-Akt. $\beta$-Actin was used as a loading control. This experiment represents one of 3. (C) HGF gene expression levels in colons from Tp/2 $2^{f / f l}$ and Tp/2/MFko mice on days 0 and 15 after initial injection with AOM. Gene expression was normalized to $B 2 m$ levels. Data represent mean \pm SEM of 6 mice per genotype (2 mice from each of 3 individual experiments). ${ }^{\star} P<0.05,{ }^{* \star} P<0.01$. (D) Western blot analysis of colon lysates from the same mice for $p$-Met and $p$-Akt. $\beta$-Actin was used as a loading control. This experiment represents one of 3 performed. (E) Western blot analysis from epithelial cells (IECs) and stroma, isolated from wild-type and $T p / 2^{D / D}$ mice, on days 0 and 8 of the AOM/DSS experimental protocol. $\beta$-Actin was used as a loading control. This experiment represents one of 3 performed. (F) $\mathrm{Hgf}$ gene expression levels in epithelial cells (IECs) and stroma, isolated from wild-type and Tp/2D/D mice on day 8 of the AOM/ DSS experimental protocol. Gene expression was normalized to $B 2 m$ levels. Data represent mean \pm SEM of 6 mice per genotype (3 mice from each of 2 individual experiments). ${ }^{* \star} P<0.01$.

in several cases after TGF- $\beta$ treatment $(36,37)$. Interestingly, while treatment of wild-type IMFs with TGF- $\beta 3$ caused a reduction in the levels of HGF by $30 \%$, the same treatment in Tpl2-deficient cells had no inhibitory effect (Figure 6A). Moreover, induction of IMFs with other known upstream ligands of the Tpl2 pathway that are also reported to affect HGF expression, such as TNF or IL-1 $\beta$ $(38,39)$, did not lead to a differential effect in HGF production by Tpl2-deficient IMFs (Figure 6A). Tpl2 $2^{D / D}$ cells showed decreased ERK phosphorylation but normal JNK, p38 (Figure 6B), and NF-кB activation (data not shown) upon induction with IL-1 $\beta$, similar to what has been reported for mouse embryonic fibroblasts (MEFs) (16); differences in MAPK phosphorylation (Figure 6B) and NF-кB activation (data not shown) upon TNF stimulation were not significant in IMFs, indicating distinct Tpl2 signaling in this cell type. Interestingly, treatment with TGF- $\beta 3$ did not activate NF-кB (data not shown) and did not differentially affect MAPK signaling, as measured by ERK, JNK, and p38 phosphorylation (Figure 6B), indicating that Tpl 2 modulates the downregulation of HGF by TGF- $\beta 3$ independent of MAPKs or NF-кB. These results establish an important IMF-intrinsic role for Tpl2 in HGF production and its regulation by TGF- $\beta 3$.

Inhibition of c-Met activation alleviates tumor promotion caused by Tpl2 ablation. To decipher whether HGF overexpression and subsequent c-Met activation caused by Tpl 2 ablation could be causal to the phenotype observed in Tpl2-deficient mice, we inhibited in vivo the HGF-induced c-Met activation with the specific inhibitor PHA-665752 $(40,41)$. Both wild-type and $T p l 2^{D / D}$ mice were subjected to the AOM/DSS model and received from day 6 to 10 daily i.v. injections of the inhibitor at a concentration of $25 \mathrm{mg} / \mathrm{kg}$ (41). DMSO in saline was used as control. Mice were monitored for weight loss during the course of the experiment and were euthanized on day 15 , at which time the colon was resected, fixed in formalin, and paraffin embedded in order to evaluate clinical score, proliferation, and apoptosis (Figure 7A). $T p l 2^{D / D}$ mice receiving the inhibitor displayed a statistically significant reduction in body weight loss during the course of the experiment (Figure 7B). Colon length was also significantly increased in Tpl2knockout mice that were treated with the inhibitor in comparison with the DMSO-treated controls (Figure 7C). Remarkably, the HGF/c-Met inhibitor completely reversed high-grade dysplasia incidence in these mice (Figure 7, D and E). Furthermore, immunohistochemical analysis showed a significant reduction in proliferation and an increase in apoptosis in Tpl2-deficient mice receiving the inhibitor in comparison to the control group (Figure 7, D, F, and G). In conclusion, our data establish a novel intestinal fibroblast-specific role for $\mathrm{T}$ pl2 in the prevention of the HGF-driven c-Met activation and the regulation of epithelial tumorigenesis in CAC. 

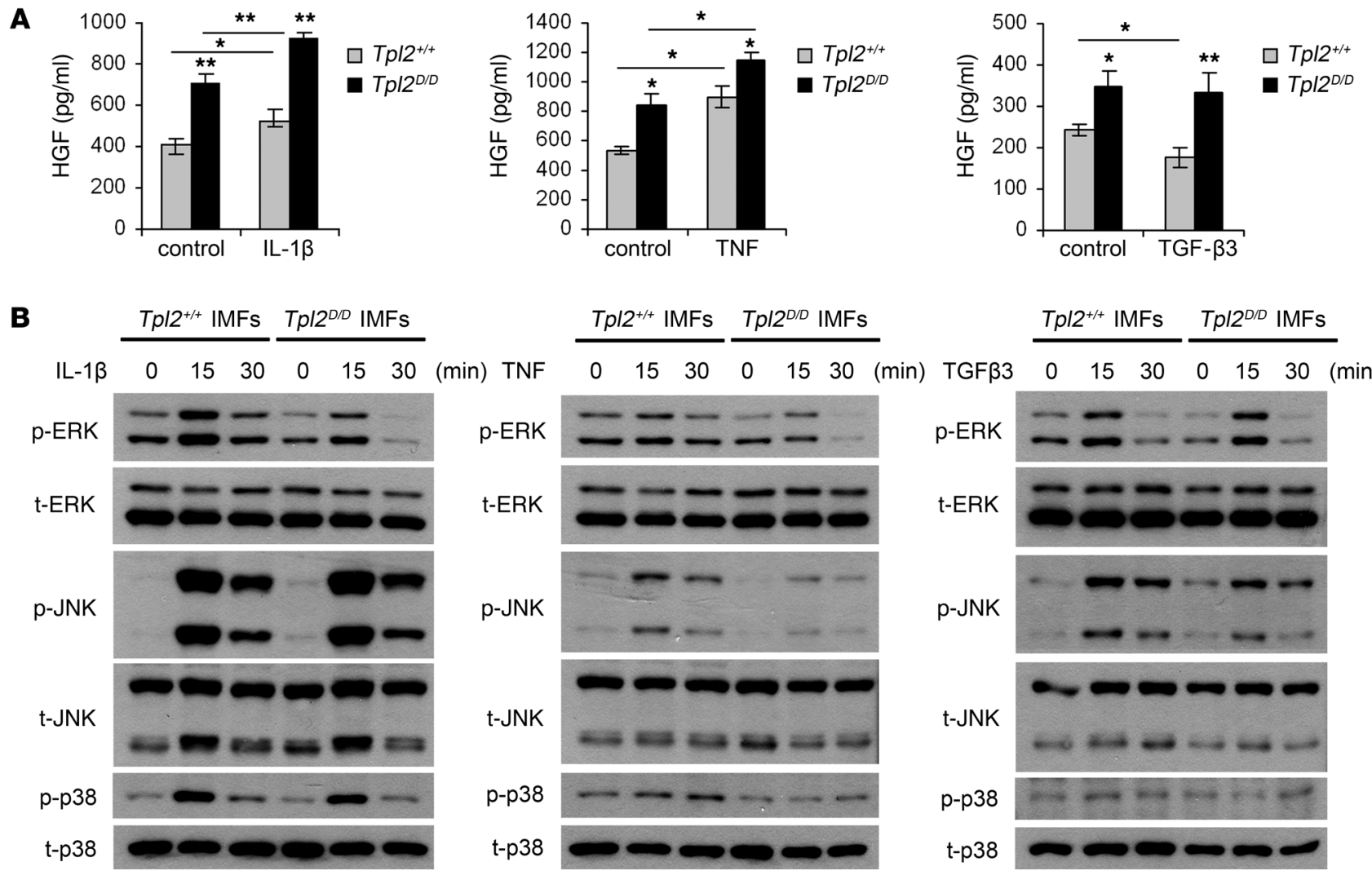

Figure 6

Tpl2 in IMFs modulates spontaneous and TGF- $\beta 3$-regulated HGF production. (A) HGF ELISA in supernatants from wild-type and Tp/2D/D primary IMF culture before and after addition of IL-1 $\beta(10 \mathrm{ng} / \mathrm{ml})$, TNF $(10 \mathrm{ng} / \mathrm{ml})$, and TGF- $\beta 3(10 \mathrm{ng} / \mathrm{ml})$ for 24 hours. Data represent mean \pm SEM of one of 3 experiments performed in triplicate. ${ }^{*} P<0.05,{ }^{* *} P<0.01$. (B) IMFs were treated with IL-1 $\beta(10 \mathrm{ng} / \mathrm{ml})$, TNF (10 ng/ml), and TGF- $\beta 3$ (10 ng/ml) for 0,15 , and 30 minutes. Cell lysates were subjected to Western blot for $p$-ERK, $p$-JNK, and p-p38. One representative experiment of 3 is shown.

\section{Discussion}

Current understanding of mechanisms underlying tumor growth and progression assigns critical functions to cells constituting the tumor microenvironment, such as endothelial cells and pericytes, tumor-infiltrating immune cells, and cancer-associated fibroblasts (42). While multiple insights have recently been gained into the role of angiogenic and inflammatory signaling in cancer, the molecular circuits by which stromal fibroblasts crosstalk with tumor cells and the microenvironment have remained largely unknown.

In the present study, we addressed potential roles played by the Tpl2 kinase in intestinal inflammation and colorectal carcinogenesis. $\mathrm{Tpl} 2$ is well known to modulate both innate and adaptive immune responses, as well as tumorigenic functions $(15,43)$ and is commonly described to respond to a variety of signals such as TLR ligands, TNF, IL-1 $\beta$, and CD40L and to activate ERK, JNK, p38, and NF-KB (17, 44-47). Considering the type of ligands to which $\mathrm{T} p 12$ is responding and their well-recognized function in inflammation and cancer $(4,48$, $49)$ including CAC $(4,5,50-52)$, it could be hypothesized that Tpl2 would serve an equally significant role. Indeed, we show in this study that in the absence of Tpl2, mice become highly susceptible to AOM/ DSS-induced colorectal carcinogenesis without, however, evidence of quantitative or qualitative changes in inflammatory infiltrates between experimental and control groups. Interestingly, administration of oncogenic AOM to Tpl2-deficient animals in the absence of the tissue-damaging and inflammatory effects of DSS did not lead to enhanced adenoma formation, which indicated that tissue damage and inflammation were required for the observed tumor-suppressive function of Tpl2 in CAC. It was surprising, therefore, to discover that Tpl2 serves a specific physiological function in CAC independent of its well-known effects in inflammation. Equally surprising was the fact that Tpl2 had no apparent function in CAC inflammation and tumorigenesis via cell-autonomous signaling in intestinal epithelial or myeloid cells. We initially observed an increase in Tpl 2 protein levels during AOM/DSS that was localized in both IECs and cells of the mucosa. Apparently, therefore, this increase in Tpl2 reflects a response to the induced changes in the overall tissue and most probably, as we show, did not play any obvious functional role. Notably, however, Tpl2 fully expressed its tumor-suppressive function via intrinsic signaling in IMFs. Recently, Tpl2 was reported to have an anti-inflammatory and tumor-suppressive role in the $A \mathrm{pC}^{\mathrm{min} /+}$ model of intestinal carcinogenesis through deregulation of IL-10 secretion and Treg generation (53). Interestingly however, despite the highlighted role for hematopoietic Tpl2 in modulating pathogenesis in this model, there was clear evidence presented in the same study of an important additional role for non-hematopoietic Tpl2 in the modulation of $A p c^{\text {min } /+}$ tumorigenesis (53). Therefore, despite well-established mechanistic differences in the pathophysiology of the $A p c^{\text {min }}$ and AOM/DSS models $(5,54,55)$, a stromal cell-intrinsic Tpl2-dependent mechanism such as the one presented in our study may be commonly contributing to the development of tumorigenesis in the two models. 
A

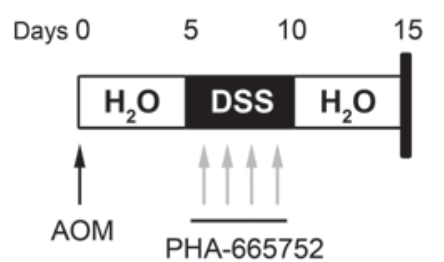

B

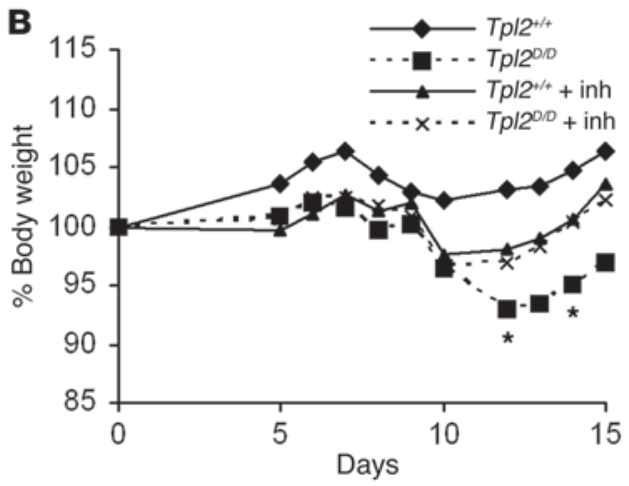

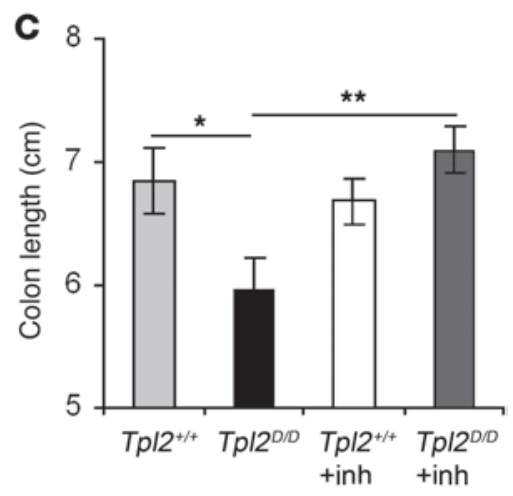

D

$T p l 2^{+/+}$

$T p / 2^{010}$

$T p / 2^{+/+}+$inh

$T p / 2^{D / D}+$ inh
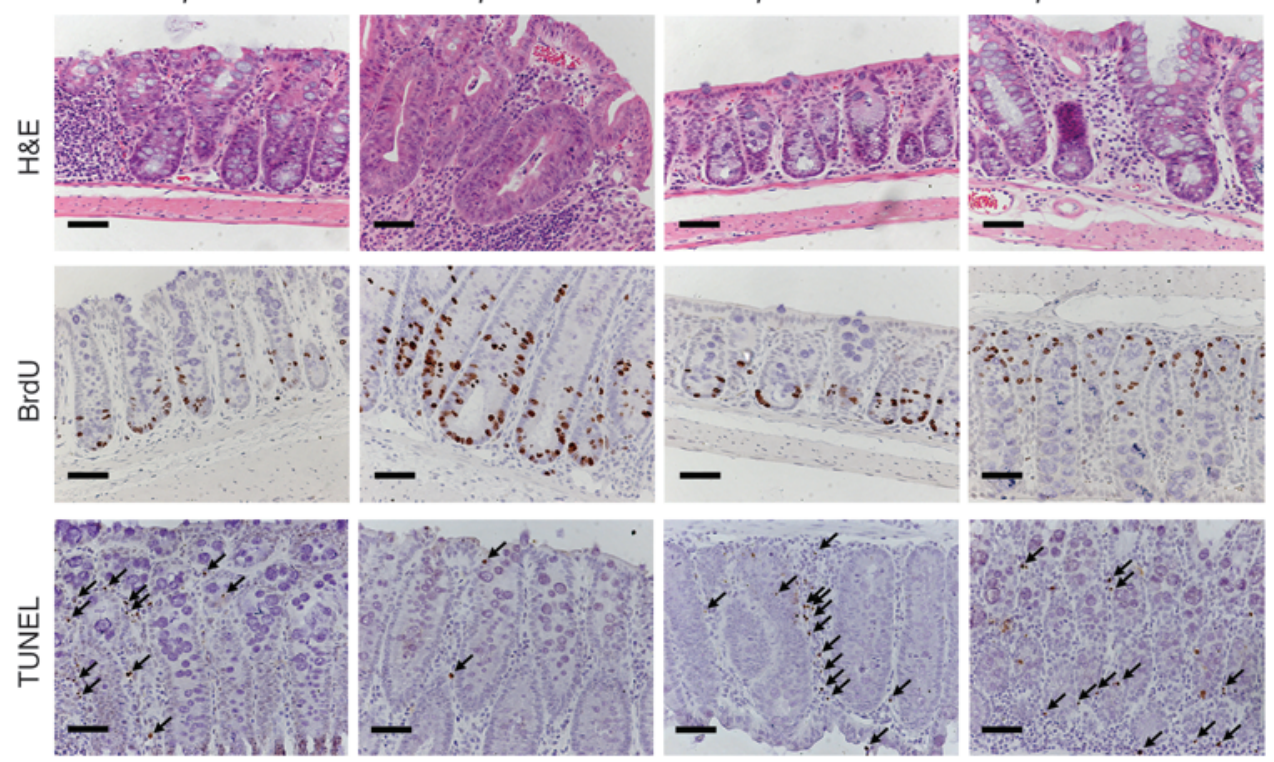

E

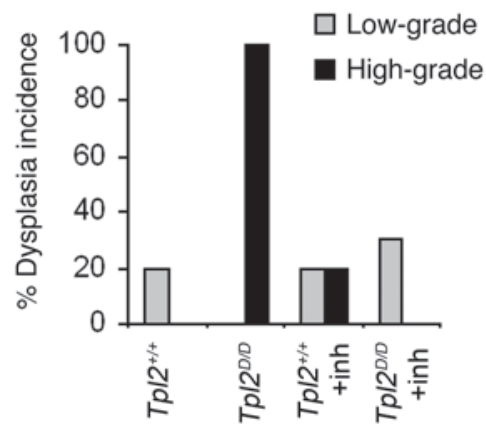

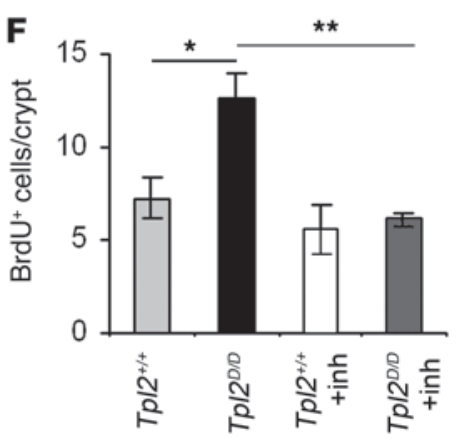

G

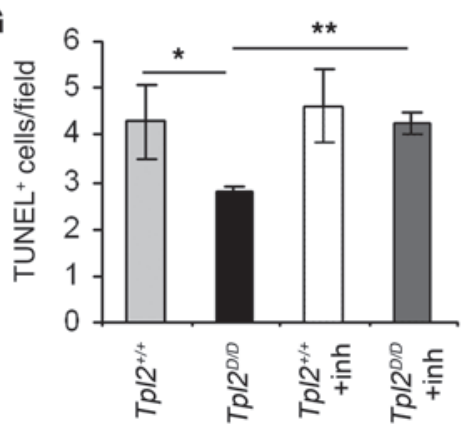

Figure 7

In vivo inhibition of HGF-driven c-Met activation blocks enhanced dysplasia in Tp/2D/D mice. (A) Diagram of PHA-665752 administration during the first 15 days of the AOM/DSS model. Tp/2D/D and wild-type littermate controls ( 5 mice per group) received 4 daily i.v. injections of PHA-665752 at a concentration of $25 \mathrm{mg} / \mathrm{kg}$ during the first DSS cycle of the CAC protocol. (B) Body weight changes were measured throughout the regime, and $(\mathbf{C})$ colon length was measured at the end of the protocol. Data represent mean \pm SEM from one of 2 experiments performed. ${ }^{*} P<0.05$, ${ }^{*} P<0.01, T p / 2^{D / D}$ mice that were injected with the inhibitor versus those that received control DMSO. $n=5$. (D) Colon tissue slides were stained with H\&E for the assessment of dysplasia index. Staining against BrdU and TUNEL-positive cells was used to determine the effect of the inhibitor in proliferation and apoptosis, respectively. Representative images from one of 2 experiments are shown. Scale bars: $50 \mu \mathrm{m}$. Arrows indicate TUNEL-positive cells (E) H\&E-stained sections were scored for dysplasia. Data represent mean \pm SEM from one of 2 experiments performed. $n=5$. Quantification of BrdU-positive cells per crypt $(\mathbf{F})$ and TUNEL-positive cells per field $(\mathbf{G})$ in colon tissue from wild-type and Tp/2/D mice with and without treatment with the c-Met inhibitor on day 15 after AOM injection. At least 20 random crypts and 10 random fields were used, respectively. Data represent mean \pm SEM. $n=6 ;{ }^{*} P<0.05,{ }^{* \star} P<0.01$. 
IMFs are an important cell type in the intestinal submucosa, exhibiting modulatory roles in both inflammation and cancer, mainly through secretion of soluble factors such as HGF, which is known to affect adjacent epithelial cells (8). In both the complete Tpl2-deficient and the IMF-specific Tpl2-knockout mice, we observed that HGF was increased early during the disease, resulting in the activation of the c-Met signaling pathway, which could also explain the increased epithelial proliferation, decreased apoptosis, and enhanced dysplasia observed early in CAC. Interestingly, HGF upregulation in the intestine could also be observed in naive Tpl2-deficient mice, indicating a physiological role for $\mathrm{T} p \mathrm{l} 2$ in controlling spontaneous HGF production in the gut. HGF is a growth factor expressed mainly by fibroblasts and is known to target cells of epithelial origin that express its receptor c-Met, leading to activation of morphogenetic, oncogenic, and metastatic responses (56). Recently, exciting work by Vermeulen et al. (11) has revealed that myofibroblast-secreted factors, in particular HGF, are able to activate clonal proliferation of cancer stem cells (CSCs) and to modulate their tumorigenicity through activation of the Wnt/ $\beta$-catenin pathway in the intestine. Therefore, HGF overexpressed mainly by fibroblast cells exerts promigratory, antiapoptotic, and mitogenic signals, leading to the oncogenic transformation of adjacent epithelial cells, although the mechanisms that control its enhanced production are not yet known. An important upstream regulator of HGF secretion in fibroblasts is TGF- $\beta$. TGF- $\beta$ RII-knockout fibroblasts exhibit increased secretion of HGF, and fibroblast-specific deletion of TGF- $\beta$ RII results in spontaneous cancer formation in the prostate and forestomach (12). Accordingly, in the present study, Tpl2-knockout fibroblasts showed a similar spontaneous increase in HGF production. Notably, treatment of IMFs from wild-type mice with TGF- $\beta 3$ caused a reduction in the levels of $\operatorname{HGF}(36,37)$, while Tpl2-deficient stromal fibroblasts were significantly less sensitive to negative regulation of HGF production by TGF- $\beta 3$, indicating that Tpl 2 promotes the tumor-suppressive signals of TGF- $\beta$. TGF- $\beta 3$ is one of the 3 TGF- $\beta$ isoforms and is mainly expressed in the gut by mesenchymal (57), goblet (58), and cancer cells (59). Interestingly, TGF- $\beta 3$ has recently been reported to induce fibroblast-specific expression of mediators of cancer stem cell maintenance (59). Given the modulatory function of Tpl 2 for the TGF- $\beta$ /HGF pathway revealed in this study, it could be hypothesized that signals known to mediate Tpl 2 function could also work downstream of TGF- $\beta$. We found that ERK, JNK and $\mathrm{p} 38$ phosphorylation in Tpl2-deficient cells was the same as in wild-type cells upon stimulation with TGF- $\beta 3$, indicating that $\mathrm{T} p \mathrm{l} 2$ regulates this pathway independent of its regular transduction of downstream MAPK and NF- $\mathrm{KB}$ signals.

In this study, administration of a small molecule inhibitor of c-Met activation in DSS/AOM-treated Tpl2-deficient mice abrogated enhanced malignant transformation in the intestine. In humans, overactivation of the c-Met pathway has been reported to occur due to genetic mutations, transcriptional upregulation, and ligand-dependent mechanisms in a variety of cancers (60). Ongoing clinical trials showing promising results are focusing on the possible therapeutic effects of $\mathrm{HGF} / \mathrm{c}-\mathrm{Met}$ inhibition in solid tumors located in several tissues, such as the lung, liver, prostate, breast, stomach, and colon (61). It is conceivable, therefore, that in addition to colorectal carcinogenesis shown in this study, a role for Tpl2 in the regulation of HGF production may apply in several other solid tumors where TGF- $\beta$ R/HGF deregulation has been causally associated with tumorigenesis, such as in mammary and squamous cell carcinomas and gastric, prostate, skin, and lung cancers $(62,63)$. The present results also indicate that caution must be exercised in the future clinical use of Tpl2 inhibition in chronic inflammatory diseases (64), pointing to a potentially enhanced susceptibility to carcinogenesis via fibroblast-specific upregulation of HGF production due to Tpl2 inhibition. In the future, detailed molecular dissection of the novel Tpl2-mediated pathway reported here may also provide clues for new rational therapies of related cancers.

\section{Methods}

Reagents. The c-Met inhibitor PHA-665752 was purchased from Sigma-Aldrich and was injected i.v. in mice at a concentration of $25 \mathrm{mg} / \mathrm{kg}$.

Mice. Generation of conditional Tpl2 knockout mice was performed using the same gene targeting strategy as the one previously described by Dumitru et al. (17). Briefly, 3 loxP sites were introduced in the Map3k8 locus, sequentially flanking a neo cassette and exon 4. Upon Cre-mediated recombination, either the floxed (Tpl2f/fl mice) or the deleted allele $\left(T p l 2^{D / D}\right.$ mice) was generated. Ella-Cre mice were used for construction of the $T p l 2^{D / D}$ mice (65). LysM-Cre mice were provided by Irmgard Forster (University of Munich, Munich, Germany). Villin-Cre transgenic mice (66) were provided by D.L. Gumucio (Department of Cell and Developmental Biology, University of Michigan Medical School, Ann Arbor, Michigan, USA). ColVI-Cre mice were previously described (33). All mice used were maintained on a mixed C57BL/6J $\times 129 \mathrm{~Sv}$ genetic background. In each experimental group, only littermate controls were used and mice caged together, according to sex. Mice were housed in the conventional unit of the animal facilities in BSRC Alexander Fleming.

Induction of CAC. Induction of CAC was performed as previously described (67). Briefly, 6- to 8-week-old mice were injected i.p. with AOM (Sigma-Aldrich) at a concentration of $10 \mathrm{mg} / \mathrm{kg}$. On day 5 after AOM injection, mice were treated with $2 \%$ DSS (MW, 36,000-50,000 Da; MP Biomedicals) in drinking water for 5 consecutive days, which was followed by 16 days of regular drinking water administration. This DSS treatment was repeated for two additional cycles, but the third one lasted 4 days. During the course of the experiment, mice were monitored for body weight, diarrhea, and macroscopic bleeding. On days 15 and 60 of the regime, colon was removed, flushed with PBS, opened longitudinally, and measured. Macroscopically visible tumors were counted. Colon sections were subsequently fixed in $10 \%$ formalin and paraffin embedded.

In other experiments, mice were injected i.p. with $10 \mathrm{mg} / \mathrm{kg}$ AOM once a week for 6 consecutive weeks. Mice were sacrificed after 18 weeks from the initial AOM injection.

Histopathology. Histology analysis was performed on H\&E colon sections. The scoring system was based on the following parameters: severity of inflammation ( 0 : rare cells in mucosa, 1: increased cells in lamina propria, 2: confluence of cells in the submucosa, 3: transmural inflammation); crypt damage (0: intact crypts, 1 : basal one-third damaged, 2 : basal twothirds damaged, 3 : only surface epithelium intact); ulceration (0: absence of ulcers, 1 : 1 or 2 ulcers, $2: 3$ or 4 ulcers, 3 : more than 4 ulcers - extensive ulceration); and percent involvement (1: $1 \%-25 \%, 2: 26 \%-50 \%, 3: 51 \%-75 \%$, 4: $76 \%-100 \%)$. Inflammation index was calculated by adding severity of inflammation and percent involvement, ranging from 0 to 7 . Tissue damage index was assessed by adding crypt damage degree, ulceration, and percent involvement, ranging from 0 to 10 . For immunohistochemistry, formalin-fixed, paraffin-embedded colon sections were stained with antibodies against F4/80 (AbD Serotec), Gr-1 (AbD Serotec), CD4 (Abcam), and Tpl2 (Santa Cruz Biotechnology Inc.). Alexa Fluor 488-anti-rat IgG (Molecular Probes) and HRP-conjugated anti-mouse IgG (Dako) were used as secondary antibodies. For Tpl2 staining, biotinylated anti-rabbit IgG (Vector Laboratories) and the Vectastain ABC kit (Vector Laboratories) were used to amplify the signal. In the case of fluorescence staining, DAPI (Santa Cruz Biotechnology Inc.) was used to stain the nuclei. In all 
other cases, signal development was performed with DAB (3,3-diaminobenzidine), and hematoxylin was used as a counterstain.

Proliferation. On day 15 after treatment with AOM/DSS, mice were injected i.p. with $100 \mathrm{mg} / \mathrm{kg}$ BrdU (Sigma-Aldrich) 2 hours prior to sacrifice. Formalin-fixed, paraffin-embedded tissues were stained for proliferating cells with a BrdU detection kit (BD Biosciences), and tissues were counterstained with hematoxylin. The number of BrdU-positive cells was quantified in intact, well-orientated crypts. At least 20 crypts were used per mouse.

Apoptosis. Colon sections from formalin-fixed, paraffin-embedded tissues were assessed for apoptotic cells using the DeadEnd Fluorometric TUNEL System (Promega) or the TACS 2 TdT-DAB In Situ Apoptosis Detection Kit (Trevigen). In the first case, DAPI (Santa Cruz Biotechnology Inc.) was used to stain the nuclei. In the second one, tissues were counterstained with hematoxylin. TUNEL-positive cells were quantified in at least 10 random fields in each slide.

Western blotting. Cells or distal colon sections were lysed in RIPA buffer, containing $1 \%$ Triton X-100, $1 \%$ sodium deoxycholate, $0.1 \%$ SDS, $150 \mathrm{mM} \mathrm{NaCl}$, $10 \mathrm{mM}$ Tris HCl, pH 7.4, 1 mM EDTA, protease inhibitors (Sigma-Aldrich), and phosphatase inhibitors (Sigma-Aldrich). Total protein content was determined using the Bradford assay (Bio-Rad), and samples were subjected to SDSPAGE and subsequently transferred to a nitrocellulose membrane (Whatman $\mathrm{GmbH}$ ). After blocking (5\% BSA, 1 hour, room temperature), membranes were stained using mouse anti-p-ERK (1:1,000; Santa Cruz Biotechnology Inc.), rabbit anti-p-JNK (1:1,000; Cell Signaling Technology), rabbit anti-pp38 (1:1,000; Cell Signaling Technology), rabbit anti-p-Met (1:1,000; Cell Signaling Technology), rabbit anti-p-Akt (1:1,000; Santa Cruz Bioechnology Inc.), rabbit anti-iNOS (1:1,000; Cell Signaling Technology), rabbit anti- $\beta$ catenin (1:5,000; Sigma-Aldrich), rabbit anti-Sox9 (1:1,000; Millipore), mouse anti-E-cadherin (1:1,000; Cell Signaling Technology), and goat anti-vimentin (1:1,000, Chemicon). Goat anti- $\beta$-actin (1:5,000; Santa Cruz Biotechnology Inc.), goat anti-ERK (1:1,000; Santa Cruz Biotechnology Inc.), mouse anti-JNK (1:1,000; Santa Cruz), and mouse anti-p38 (1:1,000; Santa Cruz Biotechnology Inc.) were used for loading control. Secondary antibodies conjugated with HRP were purchased by Vector Laboratories and used at a concentration of 1:5,000. Signal development was performed using the ECL method.

$R N A$ extraction and $q R T-P C R$. RNA was extracted from colon sections using the TRIzol method, according to the manufacturer's instructions (Invitrogen). Five micrograms of total RNA was reverse transcribed using M-MLV reverse transcriptase (Sigma-Aldrich) and oligo-dT primers (Promega) according to the manufacturer's protocol. qRT-PCR was then performed on a Chromo4 Real-Time PCR detection system (Bio-Rad) using the SYBR Green PCR Master Mix (Invitrogen), according to the manufacturer's instructions. Forward and reverse primers were added at a concentration of $0.2 \mathrm{pmol} / \mathrm{ml}$ in a final volume of $20 \mu \mathrm{l}$. The primers used are listed in Supplemental Table 1. The expression of all genes was normalized to $\beta_{2}$-microglobulin $(B 2 m)$.

Isolation of IMFs and cell culture. For in vitro assays, intestinal myofibroblast isolation from the colon was performed as described previously (68). Briefly, colons from 7- to 9-day-old pups were dissected, flushed, and extensively washed with HBSS containing antibiotics. They were then cut into 2- to 3-mm pieces and incubated with $300 \mathrm{U} / \mathrm{ml}$ Collagenase XI (Sigma-Aldrich) and $0.1 \mathrm{mg} / \mathrm{ml}$ Dispase (Roche) in DMEM for $90 \mathrm{~min}$ utes at $25^{\circ} \mathrm{C}$. This was followed by low-speed centrifugation, after which the pellet was minced and further washed with $2 \%$ sorbitol. The last wash was followed by centrifugation at $200 \mathrm{~g}$ for 5 minutes, and the pellet was plated in a 6-well plate in complete DMEM containing 1\% L-glutamine
(Gibco), 1\% non-essential amino acids (Gibco), 1\% penicillin-streptomycin (Gibco), and $10 \mathrm{ng} / \mathrm{ml}$ gentamicin (Gibco). At passage 6, cells were checked for purity using FACs analysis with the FACsCanto II (BD). Cells that were $>80 \%$ positive for CD 90.2 (BioLegend) and $<2 \%$ for $\mathrm{CD} 45$ (BD Biosciences) were used for subsequent experiments till passage 9.

For ColVI-Cre specificity, IMFs were derived from adult mouse colon according to the protocol described in Armaka et al. (33). At passage 3 , cells were $60 \%-70 \%$ positive for CD90.2 (BioLegend) and $\alpha$-SMA (Sigma-Aldrich) and $<2 \%$ for CD45 (BD Biosciences). CD90.2-positive cells were isolated by FACs sorting on a FACSVantage SE II (BD), which resulted in approximately $98 \%$ purity.

Isolation of IECs and stroma. For IEC isolation, the colon was removed, flushed with HBSS supplemented with $2 \%$ FBS and antibiotics, cut into $0.5-\mathrm{cm}$ pieces, and extensively washed. It was then incubated in $\mathrm{HBSS} / 2 \%$ FBS, containing $1 \mathrm{mM}$ EDTA and $1 \mathrm{mM}$ DTT at $37^{\circ} \mathrm{C}$ for 45 minutes. After vigorous shaking, cells released in the supernatant were layered on a discontinuous 25\%/40\% Percoll gradient (Sigma-Aldrich) and centrifuged at $600 \mathrm{~g}$ for 10 minutes, and IECs were collected from the interphase. For isolation of the stroma, incubation with EDTA was repeated until the solution was clear. The remaining tissue was then incubated with $300 \mathrm{U} / \mathrm{ml}$ Collagenase I (Sigma-Aldrich) and $0.1 \mathrm{mg} / \mathrm{ml}$ Dispase (Roche) in DMEM for 60 minutes at $37^{\circ} \mathrm{C}$. The supernatant was then passed through a cell strainer, and after low-speed centrifugation the cell pellet was used for RNA and protein extraction as described above.

Measurement of HGF levels. $2 \times 10^{4} \mathrm{CMFs}$ from wild-type and Tpl2 $2^{D / D}$ mice were plated in each well of a 96-well plate. After they were allowed to adhere, cells were serum starved for 24 hours, before treatment with $10 \mathrm{ng} / \mathrm{ml} \mathrm{TGF}$ $\beta 3$ (Sigma-Aldrich), $10 \mathrm{ng} / \mathrm{ml} \mathrm{IL-1 \beta}$ (Peprotech), or $10 \mathrm{ng} / \mathrm{ml} \mathrm{TNF}$ (provided by C. Libert, VIB, Ghent University, Ghent, Belgium) for an additional 24 hours. Supernatant was then used for measurement with a mouse HGF DuoSet ELISA System (R\&D Systems) according to the manufacturer's instructions. Cells were stained with crystal violet at the end of the experiments.

Statistics. Data are presented as mean \pm SEM. Survival curves were assessed by log-rank test (Prism software; GraphPad). Statistical significance was determined by Student's $t$ test. $P$ values less than 0.05 were considered statistically significant.

Study approval. Animal studies were approved by the Institutional Animal Care and Use Committee of BSRC Alexander Fleming.

\section{Acknowledgments}

We thank Spiros Lalos for excellent technical assistance in histopathology and Panos Athanasakis and Michalis Meletiou for technical assistance and mouse genotyping. We also thank Sofia Grammenoudi for help with flow cytometry. This work was supported by FP7 HEALTH Collaborative project INFLA-CARE, grant agreement no. 223151, and Innovative Medicines Initiative Joint Undertaking (IMI JU) Project "Be The Cure" (BTCure), grant agreement no. 115142 to $\mathrm{G}$. Kollias.

Received for publication March 20, 2012, and accepted in revised form August 30, 2012.

Address correspondence to: George Kollias, 34 Fleming Street, 16672, Vari, Greece. Phone: 30.210.9656507; Fax: 30.210.9656563; E-mail: kollias@fleming.gr.
1. Balkwill F, Mantovani A. Inflammation and cancer: back to Virchow? Lancet. 2001;357(9255):539-545.

2. Clevers $H$. At the crossroads of inflammation and cancer. Cell. 2004;118(6):671-674.

3. Terzic J, Grivennikov S, Karin E, Karin M. Inflam- mation and colon cancer. Gastroenterology. 2010;138(6):2101-2114.

4. Saleh M, Trinchieri G. Innate immune mechanisms of colitis and colitis-associated colorectal cancer. Nat Rev Immunol. 2011;11(1):9-20.
5. Salcedo R, et al. MyD88-mediated signaling prevents development of adenocarcinomas of the colon: role of interleukin 18. J Exp Med. 2010; 207(8):1625-1636.

6. Asquith M, Powrie F. An innately dangerous 
balancing act: intestinal homeostasis, inflammation, and colitis-associated cancer. J Exp Med. 2010;207(8):1573-1577.

7. Andoh A, Bamba S, Brittan M, Fujiyama Y, Wright NA. Role of intestinal subepithelial myofibroblasts in inflammation and regenerative response in the gut. Pharmacol Ther. 2007;114(1):94-106.

8. Powell DW, Pinchuk ILV, Saada JI, Chen X, Mifflin RC. Mesenchymal cells of the intestinal lamina propria. Annu Rev Physiol. 2011;73:213-237.

9. Kosinski C, et al. Gene expression patterns of human colon tops and basal crypts and BMP antagonists as intestinal stem cell niche factors. Proc Natl Acad Sci U S A. 2007;104(39):15418-15423.

10. Pinchuk IV, Mifflin RC, Saada JI, Powell DW. Intestinal mesenchymal cells. Curr Gastroenterol Rep. 2010;12(5):310-318.

11. Vermeulen L, et al. Wnt activity defines colon cancer stem cells and is regulated by the microenvironment. Nat Cell Biol. 2010;12(5):468-476.

12. Bhowmick NA, et al. TGF-beta signaling in fibroblasts modulates the oncogenic potential of adjacent epithelia. Science. 2004;303(5659):848-851.

13. Trimboli $P$, et al. Analysis of clinical, ultrasound and colour flow-Doppler characteristics in predicting malignancy in follicular thyroid neoplasms. Clin Endocrinol (Oxf). 2008;69(2):342-344.

14. Bronisz A, et al. Reprogramming of the tumour microenvironment by stromal PTEN-regulated miR-320. Nat Cell Biol. 2011;14(2):159-167.

15. Vougioukalaki M, Kanellis DC, Gkouskou K, Eliopoulos AG. Tpl2 kinase signal transduction in inflammation and cancer. Cancer Lett. 2011; 304(2):80-89.

16. Das S, et al. Tpl2/cot signals activate ERK, JNK, and NF-kappaB in a cell-type and stimulus-specific manner. J Biol Chem. 2005;280(25):23748-23757.

17. Dumitru CD, et al. TNF-alpha induction by LPS is regulated posttranscriptionally via a Tpl2/ERKdependent pathway. Cell. 2000;103(7):1071-1083.

18. Eliopoulos AG, Dumitru CD, Wang CC, Cho J, Tsichlis PN. Induction of COX-2 by LPS in macrophages is regulated by Tpl2-dependent CREB activation signals. EMBO J. 2002;21(18):4831-4840.

19. Hall JP, et al. Pharmacologic inhibition of tpl2 blocks inflammatory responses in primary human monocytes, synoviocytes, and blood. J Biol Chem. 2007;282(46):33295-33304

20. Patriotis C, Makris A, Bear SE, Tsichlis PN. Tumor progression locus $2(\mathrm{~T} \mathrm{pl}-2)$ encodes a protein kinase involved in the progression of rodent T-cell lymphomas and in T-cell activation. Proc Natl Acad Sci U S A. 1993;90(6):2251-2255.

21. Erny KM, Peli J, Lambert JF, Muller V, Diggelmann $\mathrm{H}$. Involvement of the Tpl-2/cot oncogene in MMTV tumorigenesis. Oncogene. 1996; 13(9):2015-2020.

22. Ceci JD, et al. Tpl-2 is an oncogenic kinase that is activated by carboxy-terminal truncation. Genes Dev. 1997;11(6):688-700.

23. Krcova Z, Ehrmann J, Krejci V, Eliopoulos A, Kolar Z. Tpl-2/Cot and COX-2 in breast cancer. Biomed Pap Med Fac Univ Palacky Olomouc Czech Repub. 2008;152(1):21-25

24. Sourvinos G, Tsatsanis C, Spandidos DA. Overexpression of the Tpl-2/Cot oncogene in human breast cancer. Oncogene. 1999;18(35):4968-4973.

25. Tsatsanis C, et al. Tpl2 and ERK transduce antiproliferative $\mathrm{T}$ cell receptor signals and inhibit transformation of chronically stimulated $\mathrm{T}$ cells. Proc Natl Acad Sci U S A. 2008;105(8):2987-2992.

26. Decicco-Skinner KL, Trovato EL, Simmons JK, Lepage PK, Wiest JS. Loss of tumor progression locus 2 (tpl2) enhances tumorigenesis and inflammation in two-stage skin carcinogenesis. Oncogene. 2011;30(4):389-397.

27. Ohara R, et al. Identification of the cells expressing cot proto-oncogene mRNA. J Cell Sci. 1995; 108(pt 1):97-103.

28. Kontoyiannis D, et al. Genetic dissection of the cellular pathways and signaling mechanisms in modeled tumor necrosis factor-induced Crohn'slike inflammatory bowel disease. J Exp Med. 2002;196(12):1563-1574.

29. Lawrenz M, et al. Genetic and pharmacological targeting of TPL-2 kinase ameliorates experimental colitis: a potential target for the treatment of Crohn's disease? Mucosal Immunol. 2012;5(2):129-139.

30. Skrzypczak M, et al. Modeling oncogenic signaling in colon tumors by multidirectional analyses of microarray data directed for maximization of analytical reliability. PLoS One. 2010;5(10).pii:e13091.

31. Clausen BE, Burkhardt C, Reith W, Renkawitz $\mathrm{R}$, Forster I. Conditional gene targeting in macrophages and granulocytes using LysMcre mice. Transgenic Res. 1999;8(4):265-277.

32. el Marjou F, et al. Tissue-specific and inducible Cremediated recombination in the gut epithelium. Genesis. 2004;39(3):186-193.

33. Armaka M, et al. Mesenchymal cell targeting by TNF as a common pathogenic principle in chronic inflammatory joint and intestinal diseases. $J$ Exp Med. 2008;205(2):331-337.

34. Medema JP, Vermeulen L. Microenvironmental regulation of stem cells in intestinal homeostasis and cancer. Nature. 2011;474(7351):318-326.

35. Nakamura T, Matsumoto K, Kiritoshi A, Tano Y. Induction of hepatocyte growth factor in fibroblasts by tumor-derived factors affects invasive growth of tumor cells: in vitro analysis of tumor-stromal interactions. Cancer Res. 1997;57(15):3305-3313.

36. Matsumoto K, Tajima H, Okazaki H, Nakamura T. Negative regulation of hepatocyte growth factor gene expression in human lung fibroblasts and leukemic cells by transforming growth factor-beta 1 and glucocorticoids. J Biol Chem. 1992;267(35):24917-24920.

37. Harrison P, Bradley L, Bomford A. Mechanism of regulation of HGF/SF gene expression in fibroblasts by TGF-beta1. Biochem Biophys Res Commun. 2000;271(1):203-211.

38. Tamura M, Arakaki N, Tsubouchi H, Takada H, Daikuhara Y. Enhancement of human hepatocyte growth factor production by interleukin-1 alpha and -1 beta and tumor necrosis factor-alpha by fibroblasts in culture. J Biol Chem. 1993;268(11):8140-8145.

39. Takami Y, Motoki T, Yamamoto I, Gohda E. Synergistic induction of hepatocyte growth factor in human skin fibroblasts by the inflammatory cytokines interleukin-1 and interferon-gamma. Biochem Biophys Res Commun. 2005;327(1):212-217.

40. Christensen JG, et al. A selective small molecule inhibitor of c-Met kinase inhibits c-Met-dependent phenotypes in vitro and exhibits cytoreductive antitumor activity in vivo. Cancer Res. 2003; 63(21):7345-7355.

41. Yang $Y$, et al. A selective small molecule inhibitor of c-Met, PHA-665752, reverses lung premalignancy induced by mutant K-ras. Mol Cancer Ther. 2008;7(4):952-960.

42. Hanahan D, Weinberg RA. Hallmarks of cancer: the next generation. Cell. 2011;144(5):646-674.

43. Gantke T, Sriskantharajah S, Ley SC. Regulation and function of TPL-2, an IkappaB kinaseregulated MAP kinase kinase kinase. Cell Res. 2011;21(1):131-145.

44. Eliopoulos AG, Wang CC, Dumitru CD, Tsichlis PN. Tpl2 transduces CD40 and TNF signals that activate ERK and regulates IgE induction by CD 40 . EMBO J. 2003;22(15):3855-3864.

45. Stafford MJ, Morrice NA, Peggie MW, Cohen P. Interleukin-1 stimulated activation of the COT catalytic subunit through the phosphorylation of Thr290 and Ser62. FEBS Lett. 2006;580(16):4010-4014.

46. Salmeron A, et al. Activation of MEK-1 and SEK-1 by Tpl-2 proto-oncoprotein, a novel MAP kinase kinase kinase. ЕMBO J. 1996;15(4):817-826.
47. Chiariello M, Marinissen MJ, Gutkind JS. Multiple mitogen-activated protein kinase signaling pathways connect the cot oncoprotein to the c-jun promoter and to cellular transformation. Mol Cell Biol. 2000;20(5):1747-1758.

48. Karin M, Gallagher E. TNFR signaling: ubiquitinconjugated TRAFfic signals control stop-and-go for MAPK signaling complexes. Immunol Rev. 2009;228(1):225-240.

49. Rakoff-Nahoum S, Medzhitov R. Toll-like receptors and cancer. Nat Rev Cancer. 2009;9(1):57-63.

50. Fukata M, et al. Toll-like receptor- 4 promotes the development of colitis-associated colorectal tumors. Gastroenterology. 2007;133(6):1869-1881.

51. Popivanova BK, et al. Blocking TNF-alpha in mice reduces colorectal carcinogenesis associated with chronic colitis. J Clin Invest. 2008;118(2):560-570.

52. Xiao H, et al. The Toll-interleukin-1 receptor member SIGIRR regulates colonic epithelial homeostasis, inflammation, and tumorigenesis. Immunity. 2007;26(4):461-475.

53. Serebrennikova OB, et al. Tpl2 ablation promotes intestinal inflammation and tumorigenesis in Apcmin mice by inhibiting IL-10 secretion and regulatory T-cell generation. Proc Natl Acad Sci U S A. 2012;109(18):E1082-E1091.

54. Tanaka T, et al. A novel inflammation-related mouse colon carcinogenesis model induced by azoxymethane and dextran sodium sulfate. Cancer Sci. 2003;94(11):965-973.

55. Fodde R, et al. A targeted chain-termination mutation in the mouse Apc gene results in multiple intestinal tumors. Proc Natl Acad Sci U S A. 1994;91(19):8969-8973.

56. Birchmeier C, Birchmeier W, Gherardi E, Vande Woude GF. Met, metastasis, motility and more. Nat Rev Mol Cell Biol. 2003;4(12):915-925.

57. Achyut BR, Yang L. Transforming growth factorbeta in the gastrointestinal and hepatic tumor microenvironment. Gastroenterology. 2011; 141(4):1167-1178.

58. Dunker N, Schmitt K, Schuster N, Krieglstein K. The role of transforming growth factor beta-2, beta3 in mediating apoptosis in the murine intestinal mucosa. Gastroenterology. 2002;122(5):1364-1375.

59 . Malanchi I, et al. Interactions between cancer stem cells and their niche govern metastatic colonization. Nature. 2012;481(7379):85-89.

60. Comoglio PM, Giordano S, Trusolino L. Drug development of MET inhibitors: targeting oncogene addiction and expedience. Nat Rev Drug Discov. 2008;7(6):504-516.

61. Sharma N, Adjei AA. In the clinic: ongoing clinical trials evaluating c-MET-inhibiting drugs. Ther $A d v$ Med Oncol. 2011;3(1 suppl):S37-S50.

62. Massague J. TGFbeta in cancer. Cell. 2008; $134(2): 215-230$.

63. Bierie B, Moses HL. Tumour microenvironment: TGFbeta: the molecular Jekyll and Hyde of cancer. Nat Rev Cancer. 2006;6(7):506-520.

64. George D, Salmeron A. Cot/T pl-2 protein kinase as a target for the treatment of inflammatory disease. Curr Top Med Chem. 2009;9(7):611-622.

65. Lakso M, et al. Efficient in vivo manipulation of mouse genomic sequences at the zygote stage. Proc Natl Acad Sci U S A. 1996;93(12):5860-5865.

66. Madison BB, et al. Cis elements of the villin gene control expression in restricted domains of the vertical (crypt) and horizontal (duodenum, cecum) axes of the intestine. J Biol Chem. 2002;277(36):33275-33283.

67. Neufert C, Becker C, Neurath MF. An inducible mouse model of colon carcinogenesis for the analysis of sporadic and inflammation-driven tumor progression. Nat Protoc. 2007;2(8):1998-2004.

68. Shaker A, et al. Epimorphin deletion protects mice from inflammation-induced colon carcinogenesis and alters stem cell niche myofibroblast secretion. J Clin Invest. 2010;120(6):2081-2093. 